\title{
A Distributed Network for Intensive Longitudinal Monitoring in Metastatic Triple-Negative Breast Cancer
}

\author{
C. Anthony Blau, MDª,b, ; Arturo B. Ramirez, PhD ${ }^{\mathrm{a}, \mathrm{e}}$; Sibel Blau, MD ${ }^{\mathrm{a}, \mathrm{cf}}$; Colin C. Pritchard, MD, PhD ${ }^{\mathrm{a}, \mathrm{g}}$; \\ Michael O. Dorschner, PhD ${ }^{\mathrm{a}, \mathrm{gh}}$; Stephen C. Schmechel, MD, PhD ${ }^{\mathrm{a}, \mathrm{h}}$; Timothy J. Martins, $\mathrm{PhD}^{\mathrm{b}, \mathrm{i}}$; \\ Elisabeth M. Mahen, BS ${ }^{\mathrm{a}, \mathrm{b}, \mathrm{c}}$; Kimberly A. Burton, PhD ${ }^{\mathrm{a}, \mathrm{b}, \mathrm{c}}$; Vitalina M. Komashko, PhD ${ }^{\mathrm{a}, \mathrm{j}}$; \\ Amie J. Radenbaugh, PhD ${ }^{\mathrm{a}, \mathrm{k}}$; Katy Dougherty, MT(ASCP) ${ }^{\mathrm{d}, \mathrm{g}}$; Anju Thomas, MT(ASCP) $)^{\mathrm{d}, \mathrm{g}}$; \\ Christopher P. Miller, PhD ${ }^{\mathrm{b}, \mathrm{c}}$; James Annis, BA ${ }^{\mathrm{b}, \mathrm{i}}$; Jonathan R. Fromm, MD, PhD ; Chaozhong Song, PhD ${ }^{\mathrm{a}, \mathrm{b}}$; \\ Elizabeth Chang, BSc; Kellie Howard, PhD'; Sharon Austin, BS ${ }^{1}$; Rodney A. Schmidt, MD, PhD; \\ Michael L. Linenberger, $\mathrm{MD}^{\mathrm{c}, \mathrm{d}, \mathrm{m}} ;$ Pamela S. Becker, MD, PhD ${ }^{\mathrm{a}, \mathrm{b}, \mathrm{c}, \mathrm{d}, \mathrm{m}} ;$ Francis M. Senecal, MD ${ }^{\mathrm{a}, \mathrm{c}, \mathrm{f}}$; \\ Brigham H. Mecham, PhD ${ }^{\mathrm{a}, \mathrm{j}}$; Su-In Lee, PhD ${ }^{\mathrm{n}}$; Anup Madan, PhD'; Roy Ronen, $\mathrm{PhD}^{\mathrm{a}, \mathrm{o}}$; \\ Janusz Dutkowski, PhD ${ }^{\mathrm{a}, \mathrm{o}}$; Shelly Heimfeld, PhD ${ }^{\mathrm{m}}$; Brent L. Wood, MD, PhD ${ }^{\mathrm{d}, \mathrm{gh}}$; Jackie L. Stilwell, PhD ${ }^{\mathrm{a}, \mathrm{e}}$; \\ Eric P. Kaldjian, MDa,e; David Haussler, $\mathrm{PhD}^{\mathrm{a}, \mathrm{k}}$; and Jingchun Zhu, $\mathrm{PhD}^{\mathrm{a}, \mathrm{k}}$
}

\section{Abstract}

Accelerating cancer research is expected to require new types of clinical trials. This report describes the Intensive Trial of OMics in Cancer (ITOMIC) and a participant with triple-negative breast cancer metastatic to bone, who had markedly elevated circulating tumor cells (CTCs) that were monitored 48 times over 9 months. A total of 32 researchers from 14 institutions were engaged in the patient's evaluation; 20 researchers had no prior involvement in patient care and 18 were recruited specifically for this patient. Whole-exome sequencing of 3 bone marrow samples demonstrated a novel ROS1 variant that was estimated to be present in most or all tumor cells. After an initial response to cisplatin, a hypothesis of crizotinib sensitivity was disproven. Leukapheresis followed by partial CTC enrichment allowed for the development of a differential high-throughput drug screen and demonstrated sensitivity to investigational $\mathrm{BH}$-mimetic inhibitors of $\mathrm{BCL}-2$ that could not be tested in the patient because requests to the pharmaceutical sponsors were denied. The number and size of CTC clusters correlated with clinical status and eventually death. Focusing the expertise of a distributed network of investigators on an intensively monitored patient with cancer can generate high-resolution views of the natural history of cancer and suggest new opportunities for therapy. Optimization requires access to investigational drugs. J Natl Compr Canc Netw 2016;14(1):8-17

From the aCenter for Cancer Innovation, University of Washington, Seattle, Washington; 'Institute for Stem Cell and Regenerative Medicine, University of Washington, Seattle, Washington; 'Department of Medicine/ Hematology, University of Washington, Seattle, Washington; dSeattle Cancer Care Alliance, Seattle, Washington; 'RareCyte Inc., Seattle, Washington; ${ }^{\text {} N}$ Northwest Medical Specialities, Puyallup and Tacoma, Washington; 9Department of Laboratory Medicine, University of

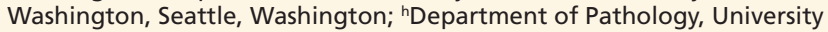
of Washington, Seattle, Washington; 'Quellos High Throughput Screening Core, Institute for Stem Cell \& Regenerative Medicine, University of Washington, Seattle, Washington; iTrialomics LLC, Seattle, Washington: kUniversity of California at Santa Cruz, Santa Cruz, California; 'Covance/ LabCorp Inc., Seattle, Washington; ${ }^{m}$ Fred Hutchinson Cancer Research Center, Seattle, Washington; nschool of Computer Science and

Engineering, University of Washington, Seattle, Washington; and 'Data4Cure Inc., La Jolla, California.

Submitted May 30, 2015; accepted for publication October 26, 2015.
Drs. Ramirez, Stilwell, and Kaldjian are employees of RareCyte; Drs. Madan, Austin, and Howard are employees of Covance/LabCorp Inc.; Drs. Komashko and Mecham are employees of Trialomics LLC; and Drs. Ronen and Dutkowski are employees of Data4Cure Inc. The remaining authors have disclosed that they have no financial interests, arrangements, affiliations, or commercial interests with the manufacturers of any products discussed in this article or their competitors. This publication was supported by the National Center for Advancing Translational Sciences of the National Institutes of Health under Award Number UL1TR000423. Funding for this work was provided by South Sound CARE, the Gary E. Milgard Family Foundation, the Pano Koumantaros Cancer Research Fund, the Washington Research Foundation, the University of Washington School of Medicine, NIDDK grant P30 DK56465 (S.H.), and private donors. Correspondence: C. Anthony Blau, MD, University of Washington, 850 Republican Street, Box 358056, Seattle, WA 98109.

E-mail: tblau@uw.edu 
A Distributed Network for Cancer

\section{Background}

In 2014, cancer in the United States claimed approximately 586,000 lives and cost $\$ 86$ billion; however, efforts to reduce these costs confront enormous inefficiencies. ${ }^{1}$ Conventional trials often require hundreds or thousands of patients to evaluate a single intervention, and many cancer treatments fail because the contextual requirements for success are not understood. ${ }^{2}$ Next-generation sequencing demonstrates that each individual's tumor contains a distinct complement of mutations, and that genomic heterogeneity extends to differences between tumor cells within each patient, ${ }^{3-14}$ motivating the development of new types of clinical trials. ${ }^{2}$ To complement the increasing number of retrospective investigations of "exceptional responders" from conventional trials, ${ }^{15-18}$ this article describes the first prospective investigation of a patient through the Intensive Trial of OMics in Cancer (ITOMIC). The ITOMIC design characterizes the molecular features of a cancer, deploys a distributed network to analyze results and predict drug susceptibilities, allows for treatment in accordance with these predictions, and aims to learn from individual patient experiences to iterate and improve over time. ${ }^{2}$ The first of these trials (ITOMIC-001) enrolls patients with metastatic triple-negative breast cancer (TNBC) (ClinicalTrials.gov identifier: NCT01957514). Results from the first patient reported from this trial exemplify the approach.

\section{Methods}

\section{Study Design}

Patients eligible for ITOMIC-001 have metastatic TNBC, are platinum-naïve, and are scheduled to receive cisplatin, which has been associated with a response rate of approximately 33\% in metastatic TNBC. ${ }^{19}$ This trial design places patients on a common therapeutic path while uncoupling initial therapy (which is typically required urgently) from the analysis of their tumors. Study participants are enrolled from either an academic site (the Seattle Cancer Care Alliance) or a private oncology practice (Northwest Medical Specialties, Tacoma and Puyallup, WA). Multiple tumor samplings are performed before treatment with cisplatin, following completion of cisplatin treatment, and following subsequent therapies. Samples are selected for whole-exome sequencing (WES), RNA-sequencing (RNA-Seq), and deep sequencing of a panel of approximately 200 cancer-associated genes (UW-OncoPlex). Oversight is provided by a Data Safety and Monitoring Board.

\section{Analysis by a Distributed Network of Investigators}

Sequencing is performed at the University of Washington (UW) in the Departments of Pathology (WES and RNA-Seq) and Laboratory Medicine (UW-OncoPlex). WES and UW-OncoPlex are performed in accordance with Clinical Laboratory Improvement Amendments (CLIA) standards. De-identified results from WES and RNA-Seq are placed on a Webbased server (DNAnexus, Mountain View, CA) to enable rapid analysis by a distributed network of investigators. Initial evaluation is focused on identifying somatic variants that may predict responsiveness to a drug, and results are intended to be available by the time the patient has completed cisplatin (due to either treatment toxicity or disease resistance). Somatic variants are identified by scientists at the University of California Santa Cruz, UW, Data4Cure (La Jolla, CA), and Trialomics (Seattle, WA). Results across platforms and across laboratories are compared during a conference call before a meeting of the ITOMIC Tumor Board.

The ITOMIC Tumor Board comprises clinicians and scientists from multiple different institutions attending in person or remotely. Attendance is open; experts with domain knowledge relevant to a given subject's findings are recruited ad hoc. A report describing results and potential therapies is provided to the patient's oncologist. Potential therapies are prioritized according to their conceptual appeal and accessibility. Upon request, the UW Center for Cancer Innovation offers assistance to access the recommended treatments.

\section{Circulating Tumor Cell Assays}

Assessments of circulating tumor cells (CTCs) were performed as described. ${ }^{20}$

\section{Differential High-Throughput Drug Screen}

The drug sensitivity profiles of CTC-enriched and CTC-depleted cells from CliniMACS processing were compared in a high-throughput screen of 160 approved and investigational oncology drugs currently being tested in acute myeloid leukemia at UW (ClinicalTrials.gov identifier: NCT01872819). 
Blau et al

CTC-enriched and CTC-depleted cell fractions were seeded into non-tissue culture-treated 384well plates. Compounds were added 12 hours later, and after 72 hours viability was assessed. Resulting dose curves were fitted using IDBS's XLfit and to a 4-parameter logistic dose response model. For highcontent imaging, cells were incubated for 28 hours with compounds, fixed with paraformaldehyde, and stained with M30 CytoDEATH (Roche, Nacka, Sweden). Imaging was performed using an IN Cell Analyzer 2000 (GE). High-throughput drug screening was also performed in 20 TNBC cell lines.

\section{Case Presentation}

The patient was a 56-year-old woman with metastatic TNBC. In the summer of 2007 she noted a lump in her right breast. Biopsy revealed an infiltrating carcinoma, estrogen receptor (ER) $3+$, progesterone receptor (PR) 1+, HER2-, and the following month she underwent a right mastectomy with axillary lymph node dissection. Pathology revealed a $5.5-\mathrm{cm}$ infiltrating carcinoma with lobular features, lymphovascular invasion with negative margins, and metastatic involvement in 20 of 25 lymph nodes (stage pT3pN3M0). She received 6 cycles of docetaxel, doxorubicin, and cyclophosphamide followed by radiation, bilateral oophorectomies, and anastrozole. Routine follow-up in the summer of 2013 detected an elevated CA 15-3 (110 U/mL, normal range $<30 \mathrm{U} / \mathrm{mL}$ ) and a low platelet count $(84,000$ per $\mathrm{mcL}$, normal $>140,000$ per $\mathrm{mcL})$. Imaging revealed lesions in the spine, pelvis, and sacrum consistent with metastatic disease. Anastrazole was changed to letrozole and fulvestrant. Results of a bone marrow biopsy showed breast cancer cells that lacked ER, PR, and HER2. To confirm an apparent change in the tumor's biomarker status, ${ }^{21,22} 2$ subsequent bone marrow examinations were performed and both demonstrated extensive infiltration with ER-/PR-/HER2- breast cancer cells. The patient's condition worsened; letrozole and fulvestrant were discontinued and capecitabine and weekly paclitaxel were begun. The patient's clinical status continued to decline, with progressive shortness of breath and fatigue. A CT angiogram was negative and a 5-day course of prednisone (60 mg daily) was ineffective. In the fall of 2013 she provided informed consent for enrollment in ITOMIC-001.
Over the course of the patient's participation in the study, we sought the expertise of 32 researchers from 14 different institutions (Figure 1). Among the researchers, 20 had no involvement in clinical care and 18 were recruited specifically for their expertise regarding findings from this patient. Prestudy bone marrow biopsy results (Figure 2A), declining platelet count (Figure 2B), normal coagulation profile (data not shown), and leukoerythroblastic blood smear results (Figure $2 \mathrm{C}$ ) were compatible with tumor replacement of the marrow. Multiple tumor samplings were performed to reduce the risk of inadequate specimen collection and to assess the validity and frequency of mutations across tumor cells taken from different disease sites. Following a platelet transfusion for thrombocytopenia, 5 core bone marrow

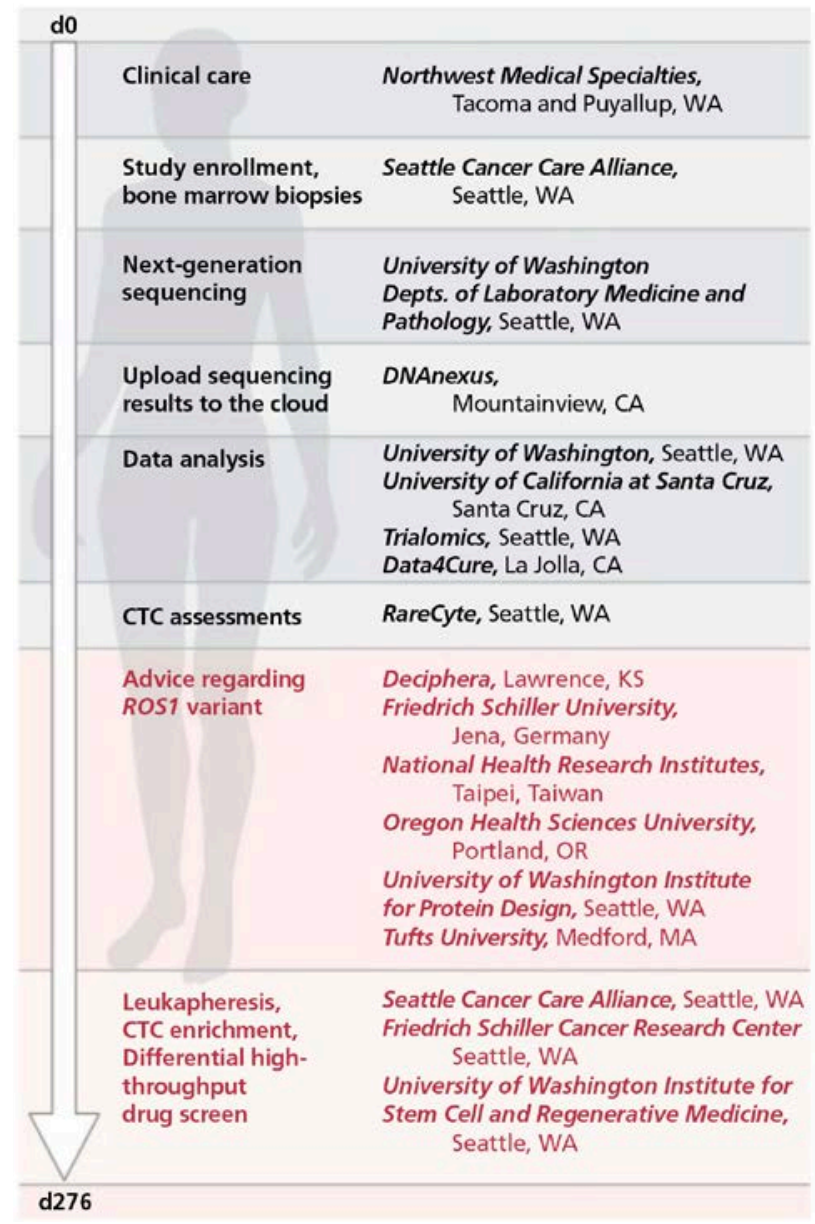

Figure 1. Assembly of a distributed network. Components in place for all patients enrolled in ITOMIC-001 are shown in black. Components added specifically for the patient presented here are shown in red. Arrow indicates timeline of the study from day 0 to the time of the patient's death on day 276.

Abbreviation: CTC, circulating tumor cell. 

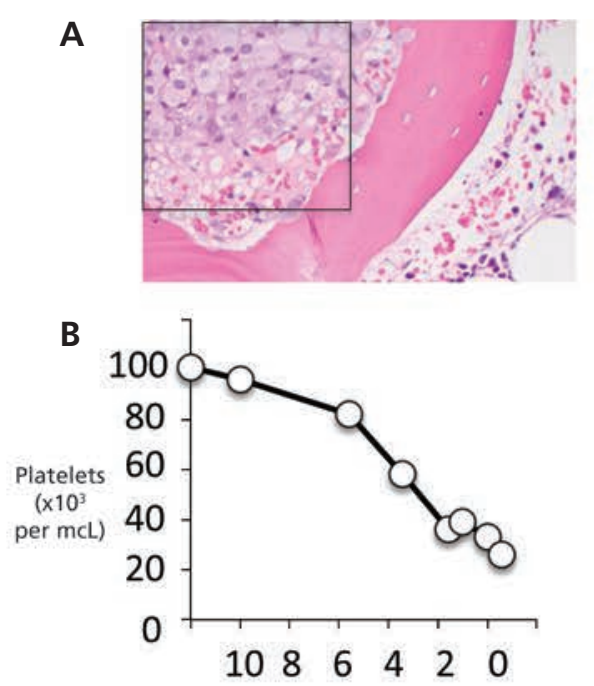

Weeks Before Enrollment

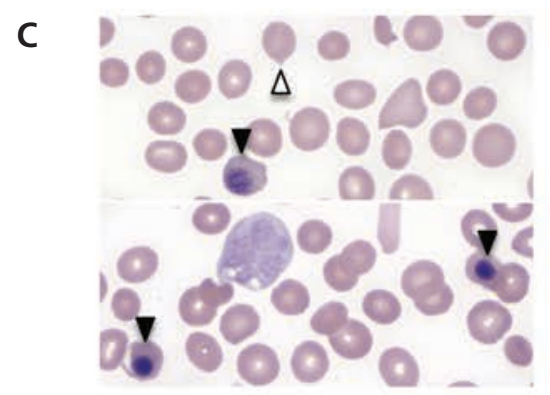

D

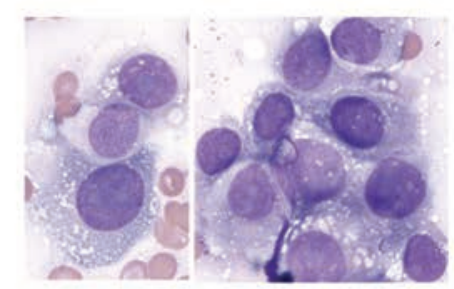

E

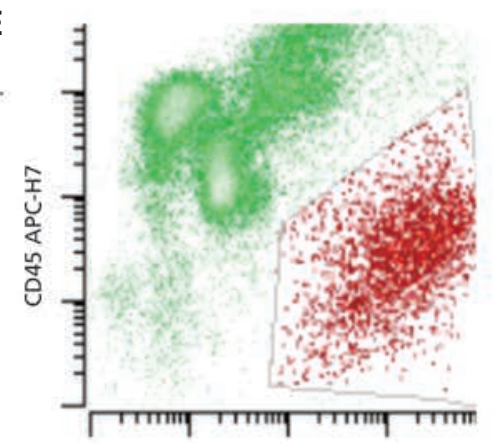

EPCAM FITC
$\mathbf{F}$

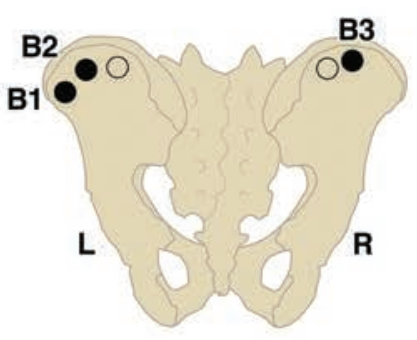

G

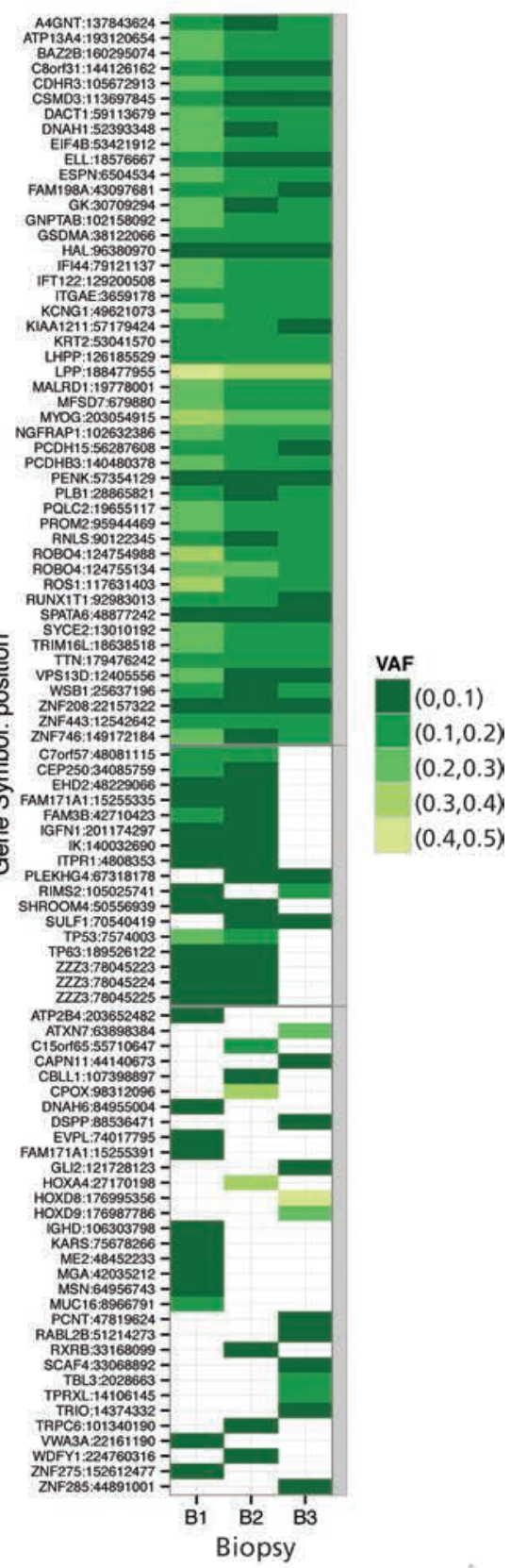

Figure 2. Clinical status at time of enrollment, bone marrow biopsies, and mutation profiles. (A) Extensive tumor involvement in a prestudy bone marrow biopsy (box). (B) Progressive decline in platelet counts in the weeks before study enrollment. (C) Nucleated red blood cells (closed arrowheads) and tear drop cells (open arrowheads), consistent with a myelophthisic process. (D) Presence of tumor cells in touch preparations from bone marrow cores obtained at study entry. (E) Representative flow cytometry profile of hematopoietic cells (green) versus tumor cells (red) in a baseline bone marrow sample. (F) Schematic depiction of biopsy sites (circles) from the left (L) and right (R) posterior iliac crest. Filled circles (designated B1, B2, and B3) indicate samples that were analyzed. (G) Schematic depiction of the mutational profile of bone marrow samples B1-B3 as determined by MuTect. Select genes are noted. Colors denote variant allele frequencies (VAF). 
Blau et al

biopsies were performed under conscious sedation (Figure 2D-F). Core samples were minced to generate cell suspensions and the 3 most abundant (B1B3) (Figure 2F) were analyzed. Estimated tumor contents ranged from 21\% to 40\%; each sample was used to perform WES, RNA-Seq, and deep sequencing of a panel of approximately 200 cancer-associated genes, ${ }^{23}$ and peripheral blood was used for germline WES and to enumerate CTCs that were found to be markedly elevated $(\approx 1500 / \mathrm{mL})$. The following day, weekly infusions of cisplatin were begun.

Low tumor purities and contamination of the peripheral blood germline sequence by CTCs complicated the detection of tumor-associated mutations (data not shown). To encompass all potentially actionable variants, we leveraged multiple samples, capture platforms, and computational algorithms (Figure 2G). All 3 mutations identified by UWOncoplex were validated by WES (data not shown). The validity of mutations was also assessed by their repeated detection across independent samples, and their attractiveness as potential therapeutic targets was assessed by their presence in most or all tumor cells as inferred from variant allele frequencies. A total of 67 single nucleotide variants and indels (including a TP53 nonsense mutation) were found in 2 of 3 samples, of which $45(67 \%)$ were detected in the spatially proximate B1 and B2 samples (Figure $2 F, G)$, consistent with regional spread of tumor subclones within the marrow. A total of 125 variants were present in all 3 bone marrow samples, and 44 missense, splice-site, and nonsense mutations were estimated to be present in most tumor cells based on their relatively high frequencies across marrow samples (Figure 2). Successful targeting of cells bearing these mutations would be expected to have a significant therapeutic benefit. Among these genes was a novel missense variant (Y2092C) of the receptor tyrosine kinase, ROS1. Other potentially actionable findings included a deletion within NF1 and a copy gain of FGFR1. ${ }^{24-26}$

\section{Response to Cisplatin}

Assessing the response to therapy in breast cancer metastatic to bone is challenging ${ }^{27}$; however, CTCs provided a sensitive and dynamic marker of the therapeutic response (Figure 3A). Seven days after the first dose of cisplatin, CTCs increased nearly 4-fold (Figure 3A), whereas CTC fragments (CD45-/EpCAM+/CK+ events lacking nuclei) increased more than 100 -fold (Figure 3B), with the ra- tio of CTC fragments to CTCs increasing from 0.05 to 1.5 ; thereafter, both CTCs and CTC fragments decreased markedly ( $>650$-fold and $>3500$-fold, respectively), mirrored by a more gradual decline in cancer antigen (CA15-3) levels (Figure 3D) and by a normalization of platelet counts (Figure 3E). These findings were consistent with a significant therapeutic effect of cisplatin, the killing and dislodgement of tumor cells in the marrow, and recovery of thrombopoiesis. Clustering increases the metastatic potential of CTCs, ${ }^{28-31}$ and cisplatin treatment was also associated with a decline in numbers of CTCs per cluster (Figure 3C).

\section{Surveying Expert Opinions Regarding a Rare ROS1 Variant and Treatment With Crizotinib}

During our analysis, attention was directed to the ROS1(Y2092C) variant, because oncogenic fusions containing the $3^{\prime}$ end of ROS1 occur in approximately $2 \%$ of non-small cell lung cancers and are associated with susceptibility to crizotinib. ${ }^{32}$ Y2092C affects a conserved residue within the ROS1 kinase domain, and a search of publicly available databases showed that ROS1(Y2092C) had been detected once previously in a 60-year-old man with non-small cell lung cancer. After a PubMed search, we contacted individuals with relevant expertise (Figure 4). All were willing to share unpublished results, and although none had tested the Y2092C variant, several theorized that it might increase ROS1 kinase activity. When the patient's case was presented at a dedicated meeting of the ITOMIC Tumor Board, members suggested consideration of crizotinib or a fibroblast growth factor receptor inhibitor.

Cisplatin was discontinued after 21 weeks because of neuropathy (Figure 3). The patient also developed headaches, and a dural biopsy demonstrated metastatic involvement with ER-/PR-/HER2 - cells. She was treated with steroids, intrathecal methotrexate, and whole-brain radiation. Restaging revealed minimal disease elsewhere, and as CTCs began to increase, we amended our protocol to allow for their collection using leukapheresis, and the leukapheresis product was used to optimize methods for CTC enrichment. The patient's oncologist elected to treat her with crizotinib, which was made available without cost through Pfizer's patient assistance program. CTCs increased sharply following the initiation of crizotinib, accompanied by a decline in platelet count and worsening performance status, and the 


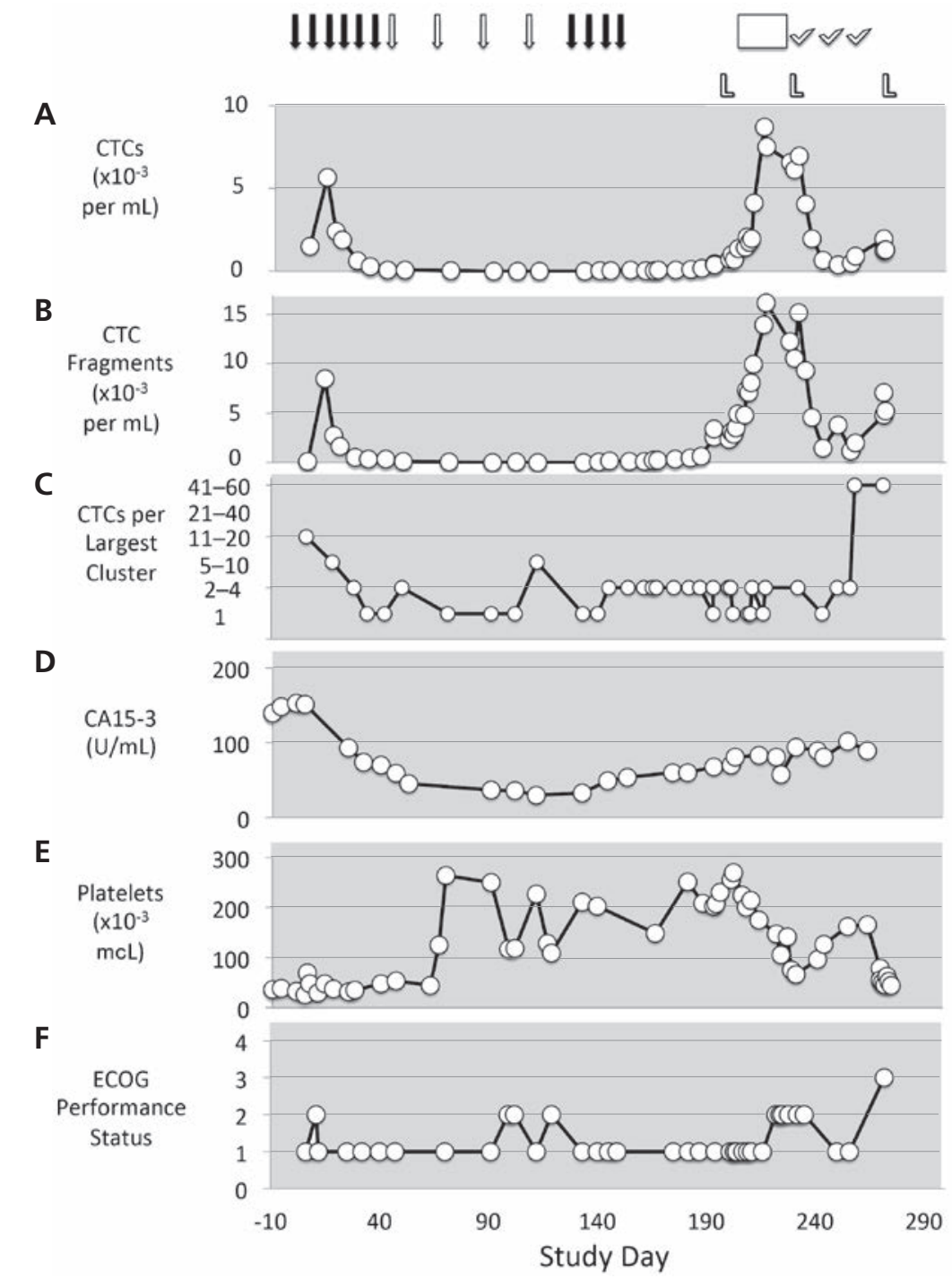

Figure 3. Dynamic changes in circulating tumor cells (CTCS) in response to therapeutic interventions. (A) CTCs; (B) CTC fragments, and (C) CTC clusters over the study period in the context of CA15-3 (D), platelet counts (E), and ECOG performance status (F). Solid arrows denote weekly cisplatin treatment. Open arrows denote cisplatin treatment every 3 weeks. L refers to leukapheresis procedures. Crizotinib treatment is noted by an open rectangle. Eribulin treatments are denoted by check marks.

drug was stopped after 3.5 weeks (Figure 3A). A second leukapheresis was performed followed by empiric treatment with the approved drug, eribulin.

\section{Leukapheresis, CTC Enrichment, and Development of a Differential High-Throughput Drug Screen}

The second leukapheresis yielded approximately 11 billion leukocytes and approximately 70 million CTCs. Following partial CTC enrichment to an estimated purity of $12 \%$, cells were used to perform a high-throughput drug screen. To interrogate a heterogeneous CTC-enriched mixture, we developed a differential drug screen, in which both cell pools obtained after magnetic bead separation-the CTC- enriched and CTC-depleted fractions-were tested and compared (Figure 5A). Among 160 drugs, only 2 exhibited preferential toxicity against the CTCenriched population: ABT-263 and ABT-737, both $\mathrm{BH} 3$-mimetic inhibitors of BCL-2 (Figure $5 \mathrm{~B}$ ). In contrast, a third BCL-2 inhibitor, obatoclax, lacked preferential antitumor activity. High-content imaging confirmed the presence of apoptotic breast cancer cells in response to ABT-263 and ABT-737 (Figure 5B), but not to obatoclax. ABT-263, ABT737, and obatoclax were further compared across 20 TNBC lines, and although responses to ABT-263 and ABT-737 were highly correlated $(r=0.89)$, there 


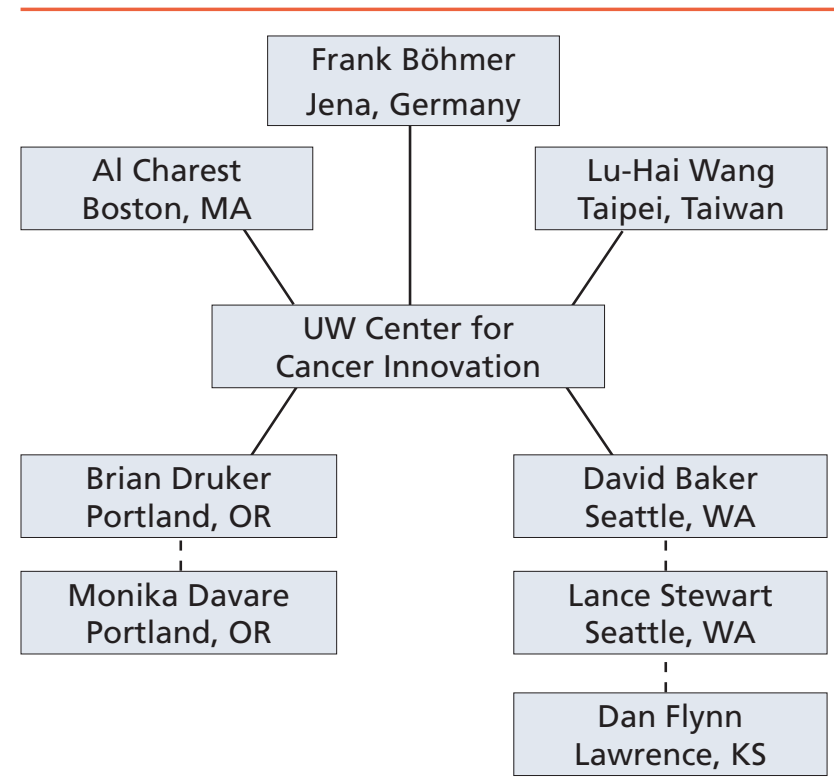

Figure 4. Experts contacted regarding the ROS1(Y2092C) variant observed in this patient. Solid lines denote primary contacts.

was no correlation between either drug versus obatoclax (Figure 5C, D). These findings are consistent with the similarities in structure and high-level affinity for BCL-2 family members for ABT-263 and ABT-737 relative to obatoclax, and with off-target effects associated with obatoclax. ${ }^{33}$

ABT-263 and ABT-737 are both investigational compounds; ABT-263 and its synthetic derivative (ABT-199) are undergoing evaluation in a number of clinical trials (ClinicalTrials.gov). Because our patient was not eligible for any of these trials, we sought to obtain access through a single-patient investigational new drug application; however, the pharmaceutical sponsors for these compounds denied our requests.

\section{Later Disease Course}

CTCs decreased sharply but only transiently in response to eribulin (Figure 3A). Repeat leukapheresis and differential high-throughput drug screening again indicated sensitivity to $\mathrm{BH}-3$ mimetic inhibitors of BCL-2 (data not shown). The following day the patient became hypoxic and her oxygenation worsened until her death 5 days later. An autopsy revealed widely disseminated intravascular carcinoma involving the lungs, epicardium, and brain, with diffuse occlusion of the arteriolar vasculature (Figure 6). Notably, although CTC levels increased moderately in concert with her clinical deterioration, a marked increase in CTC clusters was observed in the days preceding her death (Figures 6A, 3C).

\section{Discussion}

ITOMIC presents an alternative type of clinical trial that links the distributed analysis of an individual patient with cancer with interventions aimed at generating therapeutic benefit coupled with monitoring of disease response, thereby establishing a learning loop that may enable the development of more effective therapies. Contrasting the prespecified end points and outcomes inherent in most clinical trials, ITOMIC studies are flexible, leveraging opportunities presented by each patient to generate leads for future studies. Efforts to understand the significance of our patient's ROS1(Y2092C) variant exemplify several features of the approach: (1) it involves a global solicitation of expert opinion, (2) it allows these opinions to be provided back to clinicians and patients, (3) it permits predictions to be tested, (4) it provides transparency in acknowledging that predictions will frequently be proven wrong, and (5) it facilitates the reporting of outcomes. Although the clinical management of rare tumor-associated variants of uncertain significance, like ROS1(Y2092C), is controversial, ${ }^{2,34-36}$ the contexts of an incurable disease and a carefully conducted clinical trial create a setting in which informed patients can access the latest advice and technologies, while allowing their experiences to contribute to an ever-expanding body of captured knowledge. With its prospective orientation, ITOMIC can be regarded as the opposite bookend to retrospective examinations of exceptional responders from conventional trials. ${ }^{15-18}$

Results from our patient provide the most granular view of CTC dynamics yet described, integrating relationships between total CTCs, CTC fragments, and CTC clusters in the context of clinical status and response to therapy. The high CTC levels in our patient also provided an opportunity to couple leukapheresis with the ad hoc development of a differential high-throughput drug sensitivity screen. In contrast to drug sensitivity screens that rely on comparing responses across different patient samples, ${ }^{37,38}$ differential drug sensitivity profiling compares effects between targeted and nontargeted cell types within a single patient, providing an individualized in vitro assessment of therapeutic index. The differential sensitivity of CTCs to $\mathrm{BH} 3$-mimetic inhibitors of BCL-2 is noteworthy because the same class of drugs also sensitizes TNBC xenografts to chemotherapy. ${ }^{39}$ In contrast, the third BCL-2 inhibitor in 


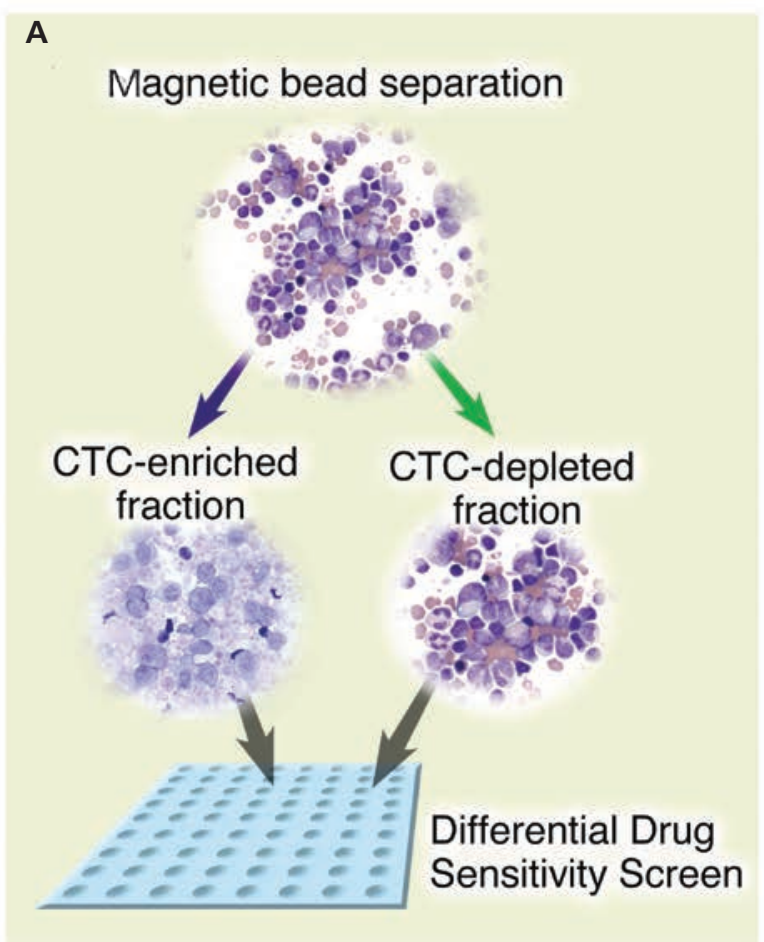

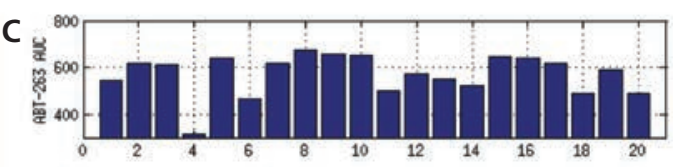
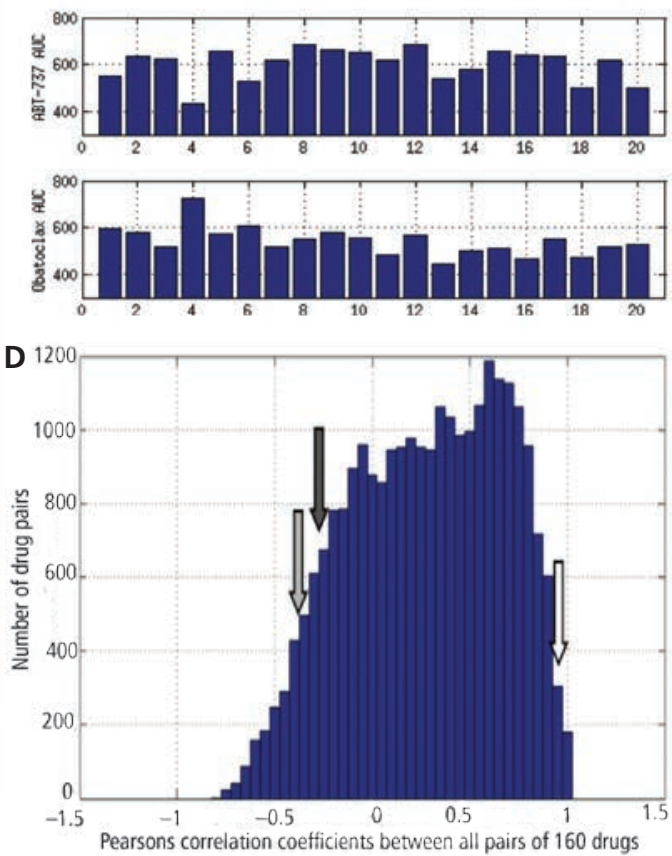

B
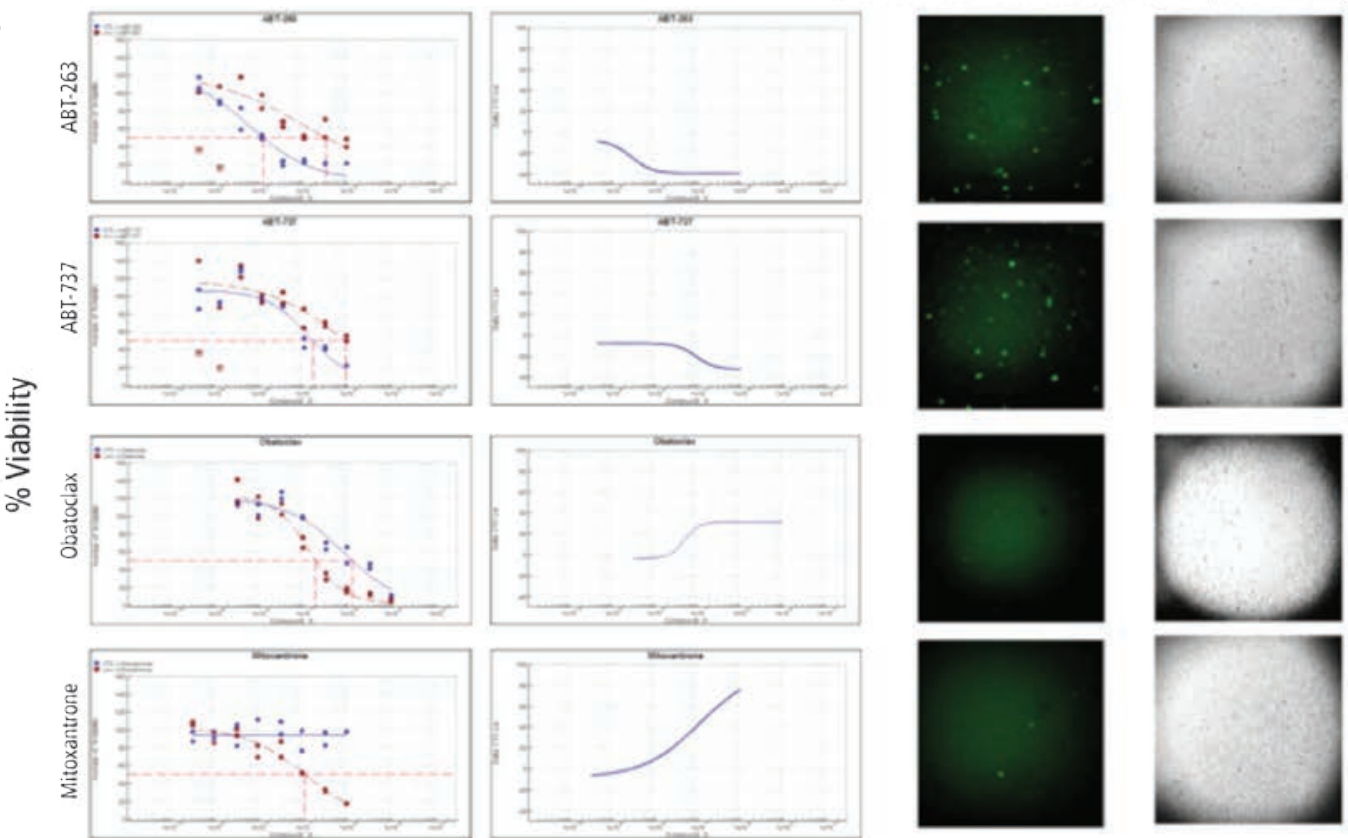

Concentration (M)
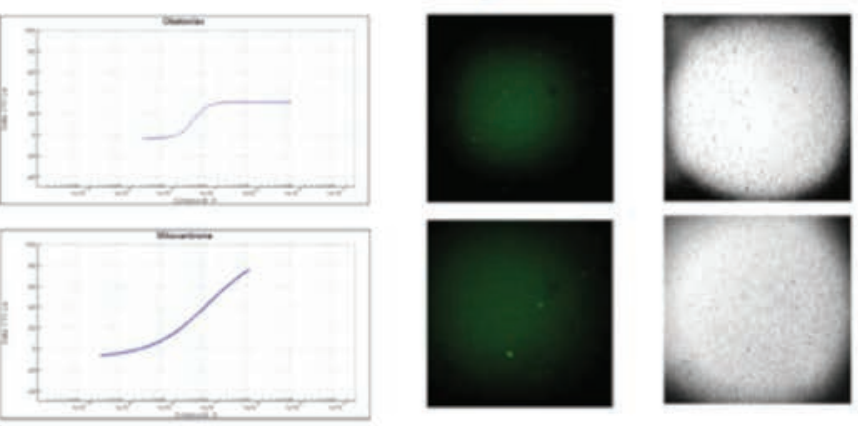

Figure 5. Development of a differential high throughput drug screen. (A) Following leukapheresis, circulating tumor cells (CTCs) were partially enriched using lineage depletion, and both the CTC-enriched and CTC-depleted cells were tested in a high-throughput screen of 160 investigational and approved oncology drugs. (B) Responses to BCL-2 inhibitors (ABT-263, ABT-737, obatoclax) and mitoxantrone (used as a positive control). Left columns show responses among CTC-enriched cells (blue lines) and CTC-depleted cells (red lines). Left middle column depicts summation of effects on CTC-enriched versus CTC-depleted cells. A declining slope indicates relatively greater potency against neoplastic cells, a rising slope indicates relatively greater potency against normal hematopoietic cells, whereas a flat line indicates similar potencies against neoplastic and hematopoietic cells. Right middle column depicts FITC-labeled, apoptotic cells detected using an antibody (M30), and the far right column depicts the corresponding bright field images. (C) Responses (indicated by area under the curve [AUC]) to ABT-263, ABT-737, and obatoclax across 20 triple-negative breast cancer (TNBC) cell lines (designated 1-20). (D) All correlations among all 160 drugs in the screen across the 20 TNBC cell lines. Open arrow shows that the correlation between ABT-263 and ABT-737 ranks in the top 3\% approximately, whereas neither drug correlates with obatoclax (gray and black arrows). 
Blau et al

our panel, obatoclax, is not an authentic-BH3 mimetic and exerts a range of off-target effects. ${ }^{33}$ Concordant responses to the BH3-mimetics (ABT-263 and ABT-737) across a panel of 20 TNBC cell lines and discordance between either drug versus obatoclax demonstrates the generalizability of this singlepatient finding. Our requests to access a $\mathrm{BH} 3-\mathrm{mi}$ metic via a single-patient clinical trial were denied, and although such efforts may not have helped our patient and could not have generated definitive findings, results would have nonetheless contributed significantly to the knowledge gathered from our patient, and may have helped inform the design of future trials for this class of drugs in patients with TNBC.
Broadening the applicability of differential drug sensitivity screening will require methods to significantly reduce the requisite number of CTC-enriched cells. For suitable patients, differential drug sensitivity profiling has theoretical advantages compared with drug sensitivity screens using ex vivo expanded CTCs, because it is faster and may be less subject to perturbations introduced by prolonged cell culture. ${ }^{40}$

By making the experiences of individual patients with cancer more informative, we aim to create a more efficient platform for learning. Realizing this goal will require further trials that bring researchers and patients together, the transfer of tissues and information rather than patients between different organizations, ${ }^{2}$ and a regulatory
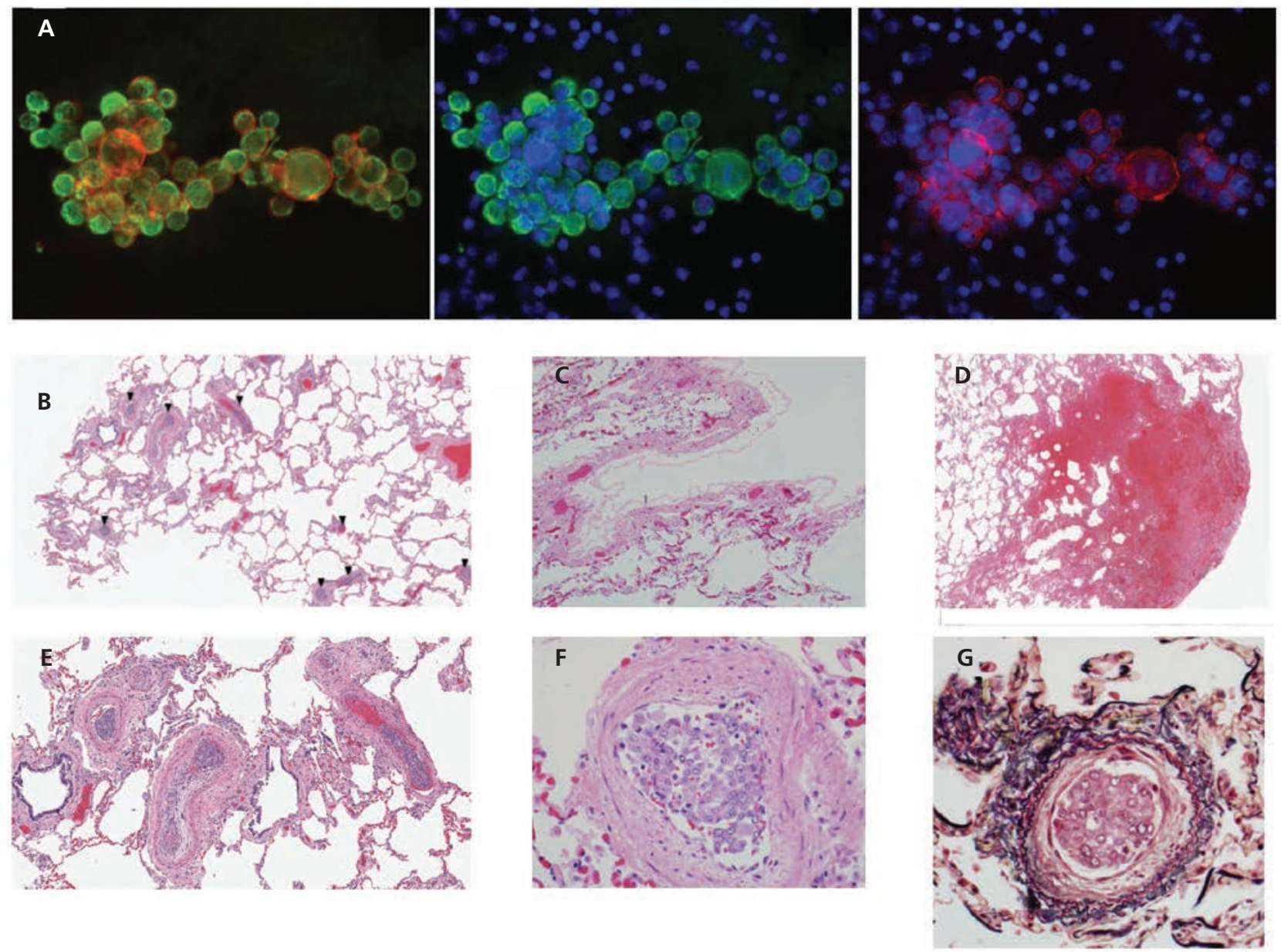

Figure 6 Circulating tumor cell (CTC) cluster late in the study and extensive tumor occlusion of the pulmonary arteriolar vasculature. (A) A CTC cluster from study day 271. Left panel shows a merged image; middle panel shows cytokeratin staining; right panel shows EpCAM staining (original magnification $\times 40)$. The blue color indicates DAPI (4',6-diamidino-2-phenylindole)-stained nuclei. (B) A low-power view of lung tissue in which multiple vessels contain clusters of dark-staining cells (arrowheads) (hematoxylin-eosin [H\&E], original magnification $x 4$ ). (C) Image shows no identified involvement of pulmonary lymphatics (H\&E, original magnification $\times 10)$. (D) Images shows a pulmonary infarct (H\&E, original magnification $\times 2)$. (E) Magnified view of Figure 6B (H\&E, original magnification x10). (F) In many of these arteries, there is near occlusion of the lumen by breast carcinoma cells, and intimal expansion by smooth muscle cells, myofibroblasts, and extracellular material (H\&E, original magnification $\times 40)$. (G) A Movat stain revealing that the vessels are arteries (containing both internal and external elastic laminae) (H\&E, original magnification $\mathrm{x} 40$ ). 
A Distributed Network for Cancer

framework that encourages thoughtful, patientcentered exploration.

\section{Acknowledgments}

The authors would like to thank the patient and her family. We also gratefully acknowledge the assistance of Michelle Flores, Melodee Smith, Sherry Littlefield, Laura Connelly-Smith, James Riddle, Wayne Monsky, Ednus Warren, Stan Riddell, Daniel Flynn, Frank Bohmer, Alain Charest, David Baker, Brian Druker, Lance Stewart, Monika Davare, Julie Gralow, Bernie McLaughlin, Jennifer Specht, VK Gadi, Paul Martin, Corinne Fligner, David Yadock, Jennifer Spokely, Krystal Malhotra, Stephanie Parker, Daniel Campton, Josh Nordberg, Nick Drovetto, Zhijun Duan, Nicole Heying, Leila Ritter, and NW BioTrust.

\section{References}

1. Scannell JW, Blanckley A, Boldon $H$, Warrington B. Diagnosing the decline in pharmaceutical $R \& D$ efficiency. Nat Rev Drug Discov 2012;11:191-200

2. Blau CA, Liakopoulou E. Can we deconstruct cancer, one patient at a time? Trends Genet 2013;29:6-10.

3. Nowell PC. The clonal evolution of tumor cell populations. Science 1976;194:23-28.

4. Dexter DL, Kowalski HM, Blazar BA, et al. Heterogeneity of tumor cells from a single mouse mammary tumor. Cancer Res 1978;38:3174-3181.

5. Stephens PJ, McBride DJ, Lin M, et al. Complex landscapes of somatic rearrangement in human breast cancer genomes. Nature 2009;462:10051010.

6. Pleasance ED, Cheetham RK, Stephens PJ, et al. A comprehensive catalogue of somatic mutations from a human cancer genome. Nature 2010;463:191-196.

7. Forbes SA, Bindal N, Bamford S, et al. COSMIC: mining complete cancer genomes in the Catalogue of Somatic Mutations in Cancer. Nucleic Acids Res 2010;39:D945-950.

8. Stephens PJ, Tarpey PS, Davies H, et al. The landscape of cancer genes and mutational processes in breast cancer. Nature 2012;486:400-404.

9. Gerlinger M, Rowan AJ, Horswell S, et al. Intratumor heterogeneity and branched evolution revealed by multiregion sequencing. $\mathrm{N}$ Engl J Med 2012;366:883-892.

10. Shah SP, Roth A, Goya R, et al. The clonal and mutational evolution spectrum of primary triple-negative breast cancers. Nature 2012;486:395399.

11. Zare $\mathrm{H}$, Wang J, Hu $\mathrm{A}$, et al. Inferring clonal composition from multiple sections of a breast cancer. PLoS Comput Biol 2014;10:e1003703.

12. Marusyk A, Almendro V, Polyak K. Intra-tumour heterogeneity: a looking glass for cancer? Nat Rev Cancer 2012;12:323-334.

13. Eirew P, Steif A, Khattra J, et al. Dynamics of genomic clones in breast cancer patient xenografts at single-cell resolution. Nature 2015;518:422426.

14. Gerlinger M, McGranahan N, Dewhurst SM, et al. Cancer: evolution within a lifetime. Annu Rev Genet 2014;48:215-236.

15. Iyer G, Hanrahan AJ, Milowsky MI, et al. Genome sequencing identifies a basis for everolimus sensitivity. Science 2012;338:221.

16. Wagle N, Grabiner BC, Van Allen E, et al. Response and acquired resistance to everolimus in anaplastic thyroid cancer. $N$ Engl J Med 2014;371:1426-1433.
17. Wagle N, Grabiner BC, Van Allen E, et al. Activating mTOR mutations in a patient with an extraordinary response on a phase I trial of everolimus and pazopanib. Cancer Discov 2014;4:546-553.

18. Al-Ahmadie H, Iyer G, Hohl M, et al. Synthetic lethality in ATMdeficient RAD50-mutant tumors underlies outlier response to cancer therapy. Cancer Discov 2014;4:1014-1021.

19. Isakoff SJ, Mayer EL, He L, et al. TBCRC009: a multicenter phase II clinical trial of platinum monotherapy with biomarker assessment in metastatic triple-negative breast cancer. J Clin Oncol 2015;33:1902-1909.

20. Campton DE, Ramirez AB, Nordberg JJ, et al. High-recovery visual identification and single-cell retrieval of circulating tumor cells for genomic analysis using a dual-technology platform integrated with automated immunofluorescence staining. BMC Cancer 2015;15:360.

21. Pusztai L, Viale G, Kelly CM, Hudis CA. Estrogen and HER-2 receptor discordance between primary breast cancer and metastasis. Oncologist 2010;15:1164-1268.

22. Criscitiello C, André F, Thompson AM, et al. Biopsy confirmation of metastatic sites in breast cancer patients: clinical impact and future perspectives. Breast Cancer Res. 2014;16:205.

23. Pritchard CC, Salipante SJ, Koehler K, et al. Validation and implementation of targeted capture and sequencing for the detection of actionable mutation, copy number variation, and gene rearrangement in clinical cancer specimens J Mol Diagn 2014;16:56-67.

24. Craig DW, O'Shaughnessy JA, Kiefer JA, et al. Genome and transcriptome sequencing in prospective metastatic triple-negative breast cancer uncovers therapeutic vulnerabilities. Mol Cancer Ther 2013;12:104-116.

25. Gozgit JM, Wong MJ, Moran L,et al. Ponatinib (AP24534), a multitargeted pan-FGFR inhibitor with activity in multiple FGFR-amplified or mutated cancer models. Mol Cancer Ther 2012;11:690-699.

26. Chiarini F, Evangelisti C, McCubrey JA, Martelli AM. Current treatment strategies for inhibiting mTOR in cancer. Trends Pharmacol Sci 2015;36:124-135.

27. Woolf DK, Padhani AR, Makris A. Assessing response to treatment of bone metastases from breast cancer: what should be the standard of care? Ann Oncol 2015;26:1048-1057.

28. Fidler IJ. The relationship of embolic homogeneity, number, size and viability to the incidence of experimental metastasis. Eur J Cancer 1973;9:223-227.

29. Liotta LA, Saidel MG, Kleinerman J. The significance of hematogenous tumor cell clumps in the metastatic process. Cancer Res 1976;36:889-894.

30. Hou J, Krebs MG, Lancashire L, et al. Clinical significance and molecular characteristics of circulating tumor cells and circulating tumor microemboli in patients with small-cell lung cancer. J Clin Oncol 2012;30:525-532.

31. Aceto N, Bardia A, Miyamoto DT, et al. Circulating tumor cell clusters are oligoclonal precursors of breast cancer metastasis. Cell 2014;158:1110 1122 .

32. Bergethon $\mathrm{K}$, Shaw AT, Ou SI, et al. ROS1 rearrangements define a unique molecular class of lung cancers. J Clin Oncol 2012;30:863-870.

33. Billard C. BH 3 mimetics: status of the field and new developments. Mol Cancer Ther 2013;12:1691-1700.

34. Micheel CM, Nass SJ, Omenn GS, eds. Evolution of Translational Omics: Lessons Learned and the Path Forward. Washington, DC: The National Academies Press; 2012.

35. Van Allen EM, Wagle N, Levy MA. Clinical analysis and interpretation of cancer genome data. J Clin Oncol 2013;31:1825-1833.

36. Kurzrock R, Kantarjian H, Stewart DJ. A cancer trial scandal and its regulatory backlash. Nat Biotechnol 2014;32:27-31.

37. Pemovska T, Kontro M, Yadav B, et al. Individualized systems medicine strategy to tailor treatments for patients with chemorefractory acute myeloid leukemia. Cancer Discov 2013;3:1416-1429.

38. Tyner JW, Yang WF, Bankhead A, et al. Kinase pathway dependence in primary human leukemias determined by rapid inhibitor screening. Cancer Res 2013;73:285-296.

39. Oakes SR, Vaillant F, Lim E, et al. Sensitization of BCL-2expressing breast tumors to chemotherapy by the $\mathrm{BH} 3$ mimetic ABT-737. Proc Natl Acad Sci USA 2012;109:2766-2771.

40. Yu M, Bardia A, Aceto N, et al. Cancer therapy. Ex vivo culture of circulating breast tumor cells for individualized testing of drug susceptibility. Science 2014;345:216-220. 